\section{Black lymph node in tattoo: an easy pitfall}

\section{Editor}

Tattoo is one of the most popular body arts in the world. It may be considered as a sign of belonging to a particular community as well as an expression of masculinity. However, it may also have a mere aesthetic purpose. In the past, whereas tattooing was common for male military personnel, bourgeois society spurned it. Nowadays, it is estimated that more than $24 \%$ of American adults have at least a tattoo. ${ }^{1}$ It is trending so tattooing is increasing social acceptability. The practice is equally common in men and women.

In Italy, this phenomenon is less extensive than in America. However, it is definitely on the rise if we compare it with the past. Tattooing is becoming extremely popular with adolescents. $^{2}$

The process of tattooing involves the repetitive piercing of the skin with ink-filled needles, which results in a permanent imprint of a design. Azo pigments are frequently used for tattooing because of their colour intensity and longevity. These azo pigments are primarily manufactured for other purposes such as printing, painting of cars and staining of various consumer products. These pigments usually contain titanium dioxide for lightening the shade, precursors and by-products of pigment synthesis, as well as diluents that are used for pigment suspension. The reaction of the skin to tattooing, the act of repeatedly tapping an ink-filled needle into the epidermis and upper 1-mm to $2-\mathrm{mm}$ of the dermis, is identical to that of minor skin injury. ${ }^{3}$

Histological evaluation of tattooed skin shows acute inflammatory reaction immediately, and within a few days macrophages phagocytize the pigment, migrate to the regional lymph nodes, and cause varying degrees of inflammatory reaction.

The presence of enlarged and black lymph nodes secondary to reaction to tattoo pigment recently has become significant in the malignant melanoma literature, where the presence of enlarged pigmented lymph nodes has led to the erroneous presumption of recurrence or metastasis. ${ }^{2}$

We describe a 21 years old woman presented to our attention for a swelling in the right inguinal region, present for about a month. Surgery consultation diagnosed inguinal hernia. During inguinal hernia's surgery, the presence of black multiple lymph nodes of increased size was noted. Therefore, a lymph node was removed for performing histological examination. Past medical history of patient was negative. Clinical examination did not evidence any atypical cutaneous lesions. Only a tattoo in right lumbar region was noticed.

On histology, the specimen of the lymph measured $1.5 \times 1 \times 0.8 \mathrm{~cm}$. Microscopic examination of the routine haematoxylin and eosin sections of the lymph node showed preservation of architecture with chronic reactive lymphadenitis. There are numerous macrophages within the lymph node containing pigment: black carbon like pigment, non-polarizable in the subcapsular and sinusoidal areas. (Fig. 1)

Immunohistochemical staining for S100, MART-1 and HMB45 shows no evidence of metastatic melanoma.

The potential migration of tattoo pigments in the draining lymph nodes was already suspected by the end of the XIXth century, however, the exact mechanism of pigment transport is not completely understood.

Recently, the SKH-1 mouse model, used for tattooing studies, was showed to present a rapid pigmentation of the draining lymph nodes of the tattooed area after the procedure. ${ }^{4}$

Tattoo pigments may be responsible for acute or chronic, transient or permanent palpable lymph nodes, that may raise concerns for tumoral or infective process. Since 1996, numerous articles have reported the occurrence of black lymph nodes and the potential risk related of mistaking such lymph nodes with a metastatic melanoma. On the basis of the presence of pigment and the physiopathological reaction that occurs in the dermis,

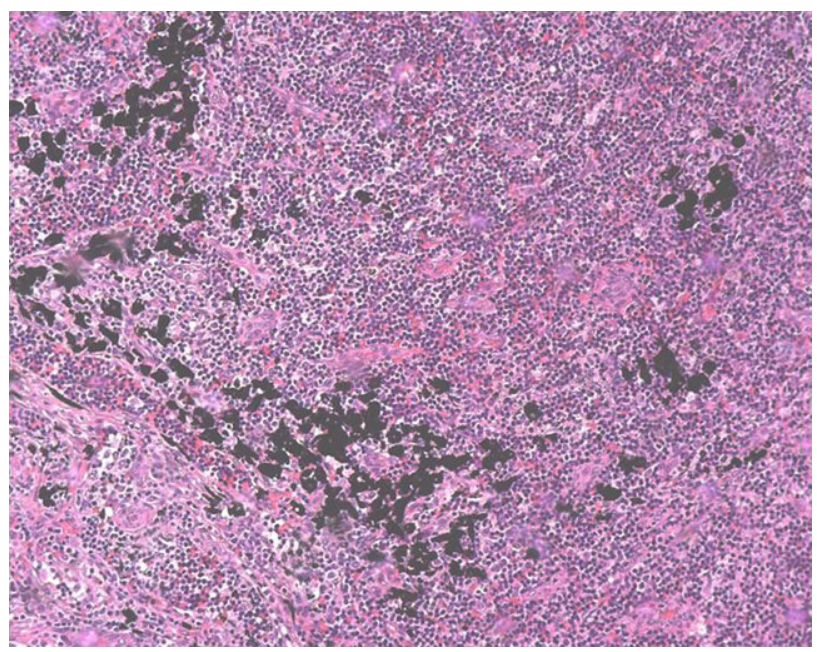

Figure 1 Haematoxylin and eosin stained section of the excised lymph node. The black pigment located in the sinuse $(H \& E \times 200)$. 
we think that every tattooed individual may have tattoo pigments in variable quantity in their regional lymph nodes. ${ }^{5}$

We suggest the surgery approach in incidental finding of a black lymph node, even in patients undergoing in surgery for other disease, in fact disadvantages caused by the removal of a lymph node are very little in relation to the possibility of a diagnosis of metastatic melanoma.

It is not the proper way to make a diagnosis of metastatic melanoma, but on the basis of the few weapons currently available to fight so aggressive cancer, this method appears lawful to our eyes.

M. Carlesimo ${ }^{1}$, G. Cortesi ${ }^{1}$, S.M. Tierno ${ }^{3}$, A. Narcisi $^{1}$, C. Abruzzese ${ }^{1}$, D. Orsini ${ }^{1}$, A. Tammaro ${ }^{1}$, F. Pulcini ${ }^{2}$, G. Mezzetti ${ }^{3}$

${ }^{1}$ Dermatology Unit, S. Andrea Hospital, II School of Medicine, University of Rome 'Sapienza', Rome, Italy, ${ }^{2} \mathrm{UOC}$ Histopathology, University of Rome Sapienza, Rome, Italy, ${ }^{3}$ Unit Surgery, Sapienza University of Rome, S. Andrea Hospital, Rome, Italy ${ }^{*}$ Correspondence: G. Cortesi. E-mail: giorgia.cortesi@libero.it

\section{References}

1 Lande RG, Bahroo BA, Soumoff A. United States military service members and their tattoos: a descriptive study. Mil Med 2013; 178: 921-925.

2 Beavis A, Amneus M, Aoyama C, Holschneider CH. Tattoo pigment lymphadenopathy mimicking metastasis in vulvar cancer. Obstet Gynecol 2012 Aug; 120(2pt2): 442-4.

3 Jemec GBE. Comment on: Tattooing of skin results in transportation and light-induced decomposition of tattoo pigments, Exp Dermatol 2010; 19: 61-62.

4 Engel E, Vasold R, Santarelli F et al. Tattooing of skin results in transportation and light-induced decomposition of tattoo pigments e a first quantification in vivo using a mouse model. Exp Dermatol 2010; 19: 54e60.

5 Kluger N, Cohen-Valensi R, Nezri M. Black lymph nodes e and a colourful skin. Lancet 2008; 371: 1214.

DOI: $10.1111 / j \mathrm{dv} .12687$ 